\title{
Study on the Influence of Air Pressure and Temperature on PM2.5 by Multivariate Functional Linear Regression Model
}

\author{
Jinjing $Y$ ang ${ }^{1, *}$ \\ ${ }^{1}$ College of Science, N orth China U niversity of Technology, P.R.China
}

\begin{abstract}
In recent years, the Internet has developed rapidly, and we have more and more ways to collect data. We find that many data have the characteristics of functions. We can use the important method of functional data analysis to analyze these data. The basic idea of functional data analysis is to treat data with functional properties as a whole for analysis and corresponding processing. In this paper, the daily air pressure, temperature and PM 2.5 data of 49 cities with serious PM 2.5 pollution in 2017 are sorted out. We use a multivariate functional linear regression model to discuss the influence of pressure and temperature on PM 2.5 when the number of basis functions is different.
\end{abstract}

\section{Introduction}

The concept of functional data was first proposed by Ledyard R Tucker in 1958. He also proposed some methods for determining function parameters in factor analysis in "Parameter Determination of Functional Relationships in Factor Analysis" [1]. In 1982, J.O. Ramsay put forward the main views and method systems of functional data analysis in "When the data are functions", and demonstrated some basic theories of functional data analysis with mathematical knowledge. In 1991, J.O. Ramsay and C.J. Dalzellz formally proposed the concept and system tools of functional data analysis in "Some Tools for Functional Data Analysis". These analysis tools were used in the analysis of temperature and precipitation of some Canadian weather stations [2]. In 2007, Professor Yan Mingyi introduced this method to China for the first time, and described the functional data analysis method from the aspects of thought, theory and technical methods, and made corresponding research by taking the employment situation of various service industries in Shaanxi as an example [3-4]. In 2017, Meng Yinfeng made corresponding research on the modeling method and application of functional data and principal component analysis method [5-6].

\section{Multivariate functional linear regression model}

Multivariate functional linear regression model

$$
y_{i}(t)=\sum_{j=1}^{q} z_{i j}(t) \beta_{j}(t)+\varepsilon_{i}(t)
$$

Where $i=1,2, \ldots, \mathrm{N}, \beta_{j}(t)$ is the regression coefficient function
The above model can be expressed as follows

$$
\begin{gathered}
Y(t)=Z(t) \beta(t)+\varepsilon(t) \\
\beta_{j}(t)=\sum_{k=0}^{K_{j}} b_{k j} \theta_{k j}(t)=\theta_{j}(t)^{\Theta} b_{j}, \\
\theta_{0 j}(t)=1 / \sqrt{K_{j}+1}, \theta_{2 r_{j}-1, j}(t)=\left(\sin r_{j} w t\right) / \sqrt{\left(K_{j}+1\right) / 2}, \\
\theta_{2 r_{j}, j}(t)=\left(\cos r_{j} w t\right) / \sqrt{\left(K_{j}+1\right) / 2} \cdot \mathrm{K}_{j}=2 r_{j} .
\end{gathered}
$$

The rough penalty term is defined as follows

$$
\operatorname{PEN}_{j}\left(\beta_{j}\right)=\lambda_{j} \int\left[D^{2} \beta_{j}(t)\right]^{2} d t
$$

The weighted regular fitting criterion is as follows

$$
\begin{gathered}
\operatorname{LMSSE}(\beta)=\int r(t)^{0} r(t) d t+\sum_{j=1}^{q} \lambda_{j} \int\left[D^{2} \beta_{j}(t)\right]^{2} d t \\
r(t)=Y(t)-Z(t) \beta(t)
\end{gathered}
$$

Let $f=\operatorname{LMSSE}(\beta)$. Finishing

$$
\begin{gathered}
\frac{\partial f}{\partial b_{1}}=0, \frac{\partial f}{\partial b_{2}}=0, \ldots, \frac{\partial f}{\partial b_{q}}=0 \\
R_{j}=\lambda_{j} \int \alpha_{j}(t) \alpha_{j}(t)^{\prime} d t \\
\alpha_{j}(t)=\left(0, w^{2} \theta_{1 j}(t), w^{2} \theta_{2 j}(t), \cdots, \frac{K_{j}^{2}}{4} w^{2} \theta_{K_{j}-1, j}(t), \frac{K_{j}^{2}}{4} w^{2} \theta_{K_{j}, j}(t)\right)^{\prime} \\
\Theta(t)=\left(\begin{array}{cccc}
\theta_{1}(t)^{\prime} & 0 & \cdots & 0 \\
0 & \theta_{2}(t)^{\prime} & \cdots & 0 \\
\vdots & \vdots & & \vdots \\
0 & 0 & \cdots & \theta_{q}(t)^{\prime}
\end{array}\right), R=\left(\begin{array}{cccc}
R_{1} & 0 & \cdots & 0 \\
0 & R_{2} & \cdots & 0 \\
\vdots & \vdots & & \vdots \\
0 & 0 & \cdots & R_{q}
\end{array}\right) \\
\text { Organized } \\
b=\left[\int \Theta(t)^{\prime} Z(t)^{\prime} Z(t) \Theta(t) d t+R\right]^{-1}\left[\Theta(t)^{\prime} Z(t)^{\prime} Y(t) d t\right.
\end{gathered}
$$

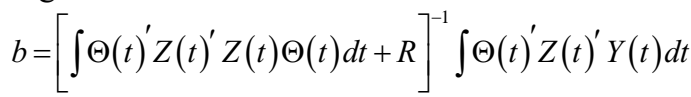

\section{Application of multivariate functional linear regression model}

\footnotetext{
*Corresponding author:2364777217@,qq.com
} 
The multivariate functional linear regression model studies the influence of multiple predictor variables on the response variable. In this article, we study the influence of the two predictors of pressure and temperature in 49 cities on PM2.5 in 2017.

The regression coefficient reflects the influence of the independent variable on the dependent variable, Its sign indicates the direction of influence. When the regression coefficient is greater than zero, it means that when the independent variable increases, the dependent variable will increase; when the regression coefficient is less than zero, it means that when the independent variable increases, the dependent variable will decrease.

We will discuss it in three categories: $K_{1}=K_{2}$, $K_{1}<K_{2}, K_{1}>K_{2}$.

The first category: $K_{1}=K_{2}$

(1) When $r_{1}=r_{2}=9$, that is, $K_{1}=K_{2}=18$, the result is as follows:
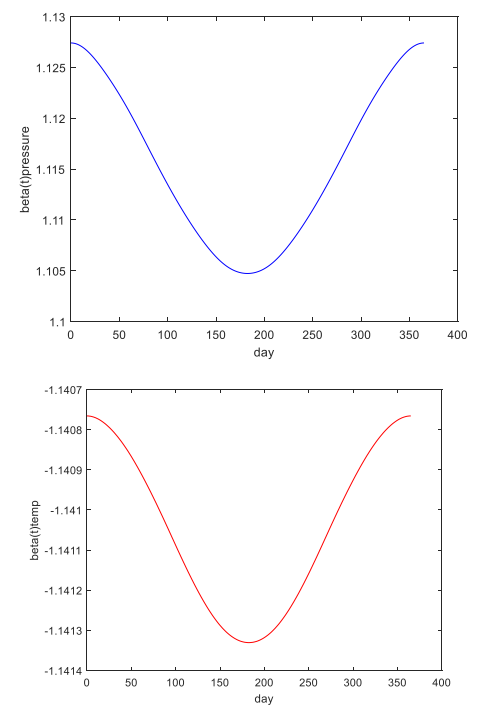

Figure 1. $K_{1}=K_{2}=18$ Two factors work together

(2) When $r_{1}=r_{2}=8$, that is, $K_{1}=K_{2}=16$, the result is as follows:
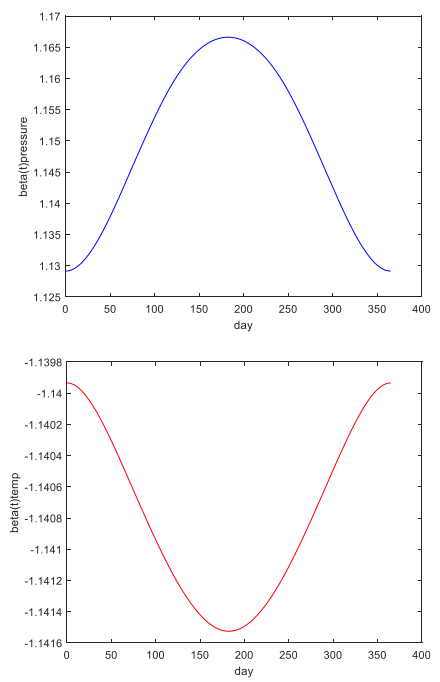

Figure 2. $K_{1}=K_{2}=16$ Two factors work together
(3) When $r_{1}=r_{2}=7$, that is, $K_{1}=K_{2}=14$, the result is as follows:
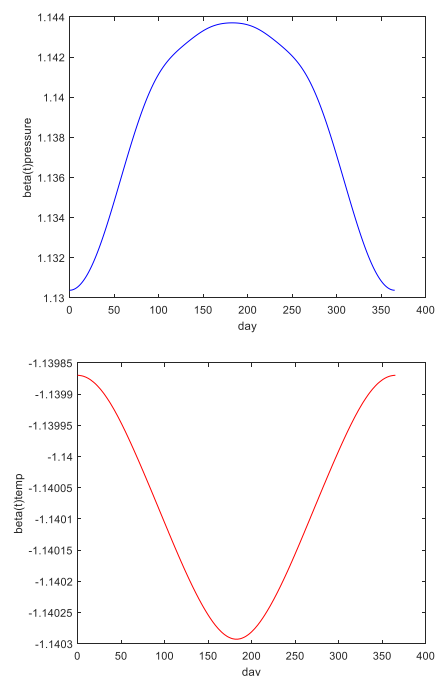

Figure 3. $K_{1}=K_{2}=14$ Two factors work together

(4) When $r_{1}=r_{2}=6$, that is, $K_{1}=K_{2}=12$, the result is as follows:
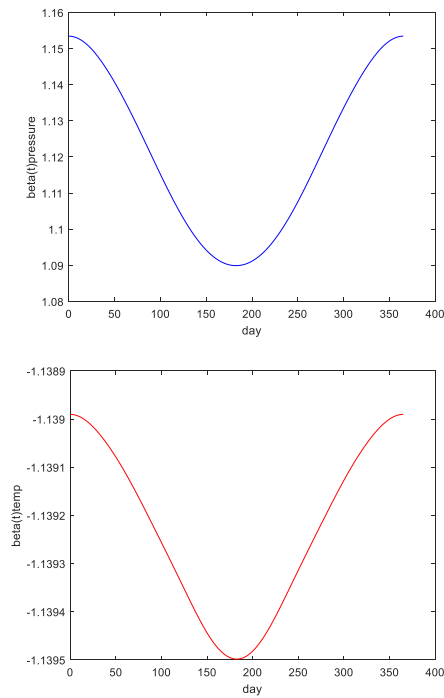

Figure 4. $K_{1}=K_{2}=12$ Two factors work together

The second category: $K_{1}<K_{2}$

(1) When $r_{1}=8, r_{2}=9$, that is, $K_{1}=16, K_{2}=18$, the result is as follows:

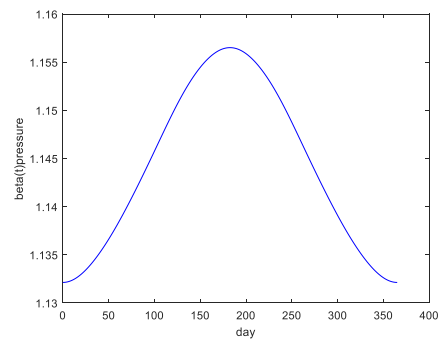




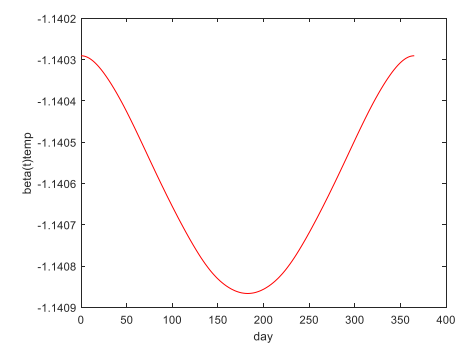

Figure 5. $K_{1}=16, K_{2}=18$ Two factors work together

(2) When $r_{1}=7, r_{2}=9$, that is, $K_{1}=14, K_{2}=18$, the result is as follows:
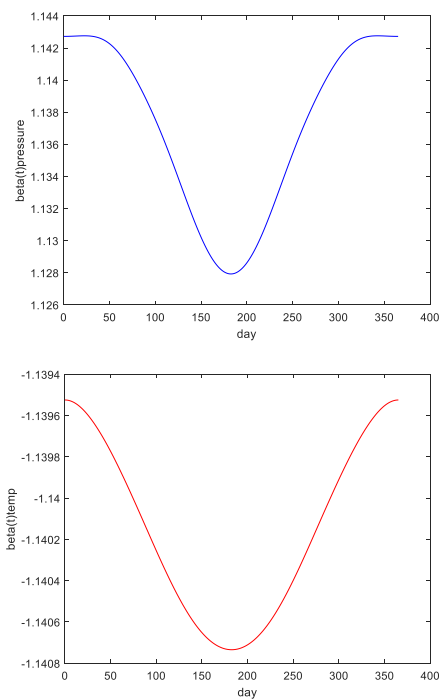

Figure 6. $K_{1}=14, K_{2}=18$ Two factors work together

(3) When $r_{1}=6, r_{2}=9$, that is, $K_{1}=12, K_{2}=18$, the result is as follows:
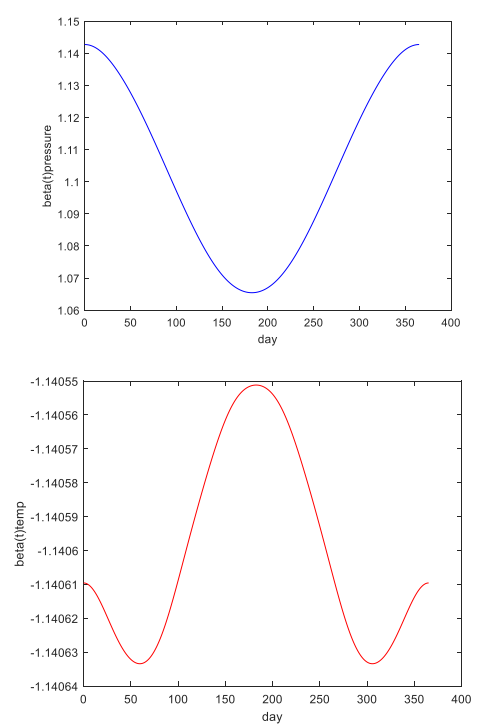

Figure 7. $K_{1}=12, K_{2}=18$ Two factors work together

(4) When $r_{1}=7, r_{2}=8$, that is, $K_{1}=14, K_{2}=16$, the result is as follows:
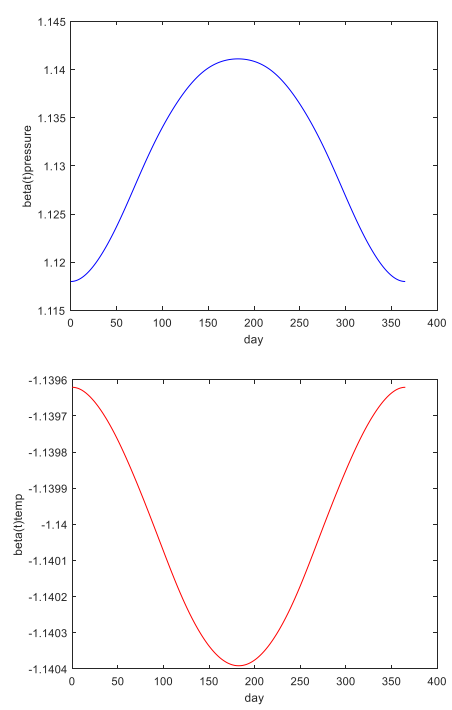

Figure 8. $K_{1}=14, K_{2}=16$ Two factors work together

(5) When $r_{1}=6, r_{2}=8$, that is, $K_{1}=12, K_{2}=16$, the result is as follows:
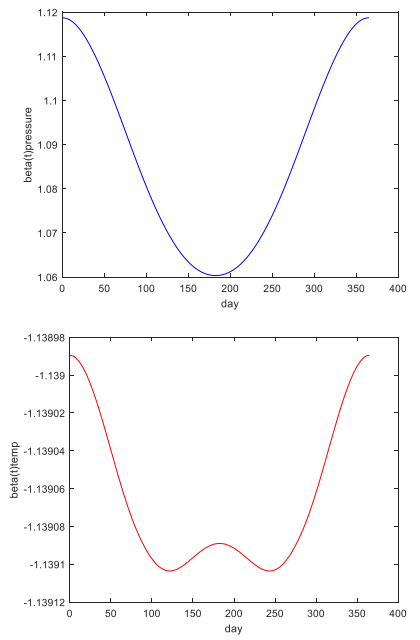

Figure 9. $K_{1}=12, K_{2}=16$ Two factors work together

(6) When $r_{1}=6, r_{2}=7$, that is, $K_{1}=12, K_{2}=14$, the result is as follows:
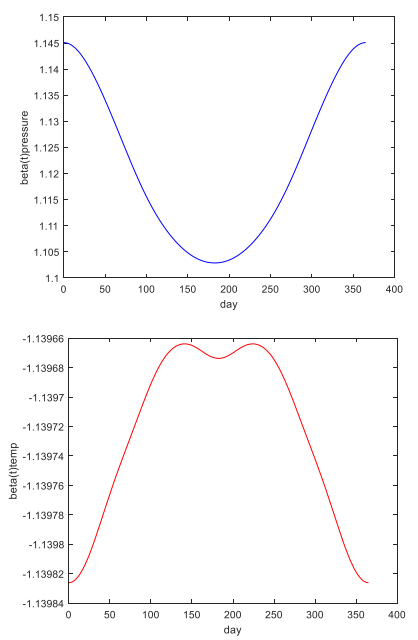

Figure 10. $K_{1}=12, K_{2}=14$ Two factors work together 
The third category: $K_{1}>K_{2}$

(1) When $r_{1}=9, r_{2}=8$, that is, $K_{1}=18, K_{2}=16$, the result is as follows:
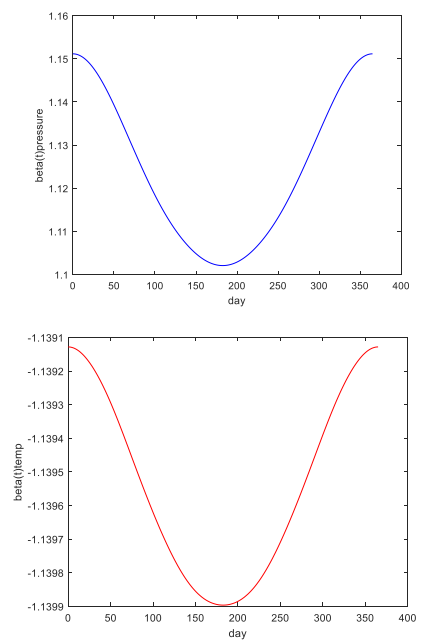

Figure 11. $K_{1}=18, K_{2}=16$ Two factors work together

(2) When $r_{1}=9, r_{2}=7$, that is, $K_{1}=18, K_{2}=14$, the result is as follows:
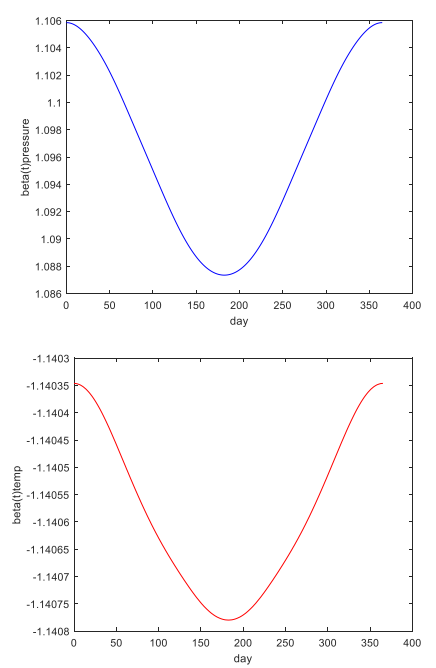

Figure 12. $K_{1}=18, K_{2}=14$ Two factors work together

(3) When $r_{1}=9, r_{2}=6$, that is, $K_{1}=18, K_{2}=12$, the result is as follows:

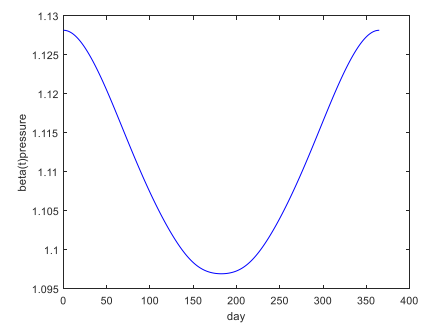

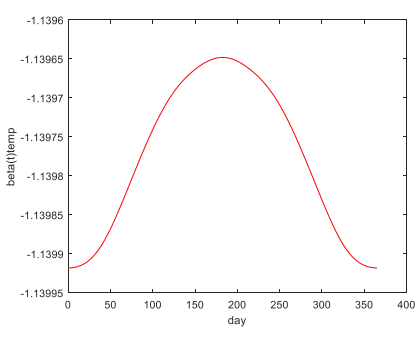

Figure 13. $K_{1}=18, K_{2}=12$ Two factors work together

(4) When $r_{1}=8, r_{2}=7$, that is, $K_{1}=16, K_{2}=14$, the result is as follows:
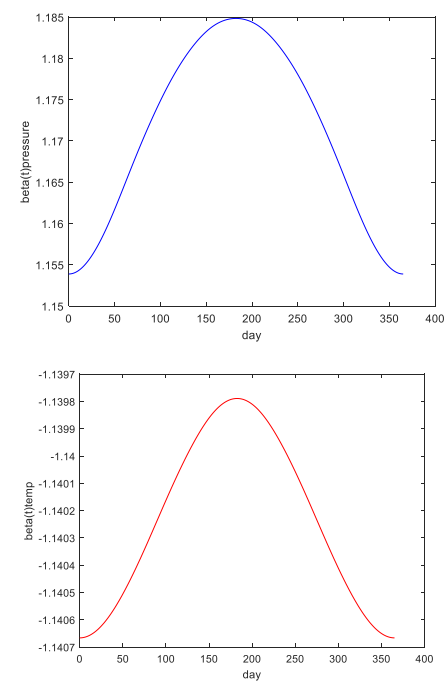

Figure 14. $K_{1}=16, K_{2}=14$ Two factors work together

(5) When $r_{1}=8, r_{2}=6$, that is, $K_{1}=16, K_{2}=12$, the result is as follows:
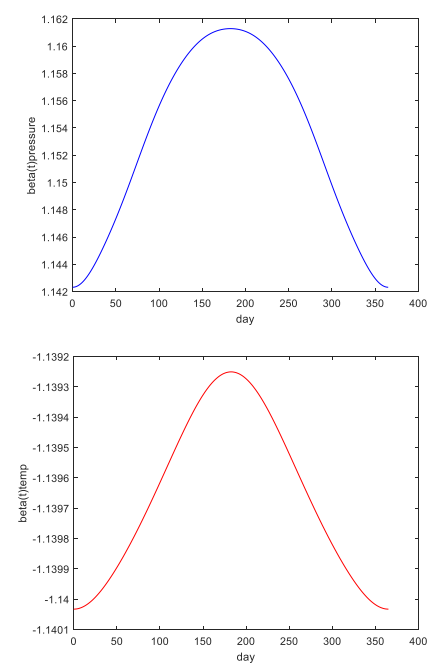

Figure 15. $K_{1}=16, K_{2}=12$ Two factors work together

(6) When $r_{1}=7, r_{2}=6$, that is, $K_{1}=14, K_{2}=12$, the result is as follows: 

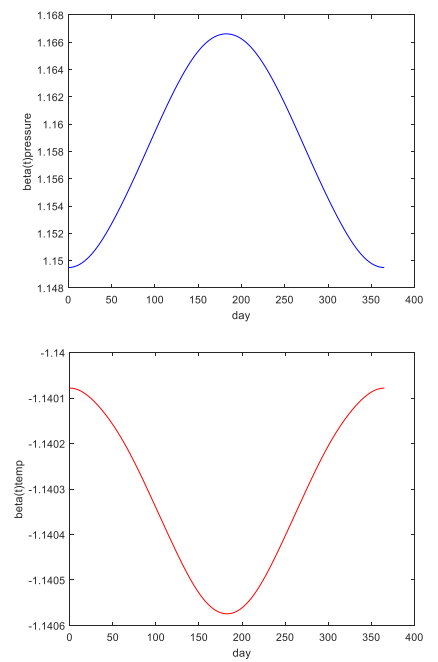

Figure 16. $K_{1}=14, K_{2}=12$ Two factors work together

\section{Conclusion}

This paper collects daily air pressure, temperature, and PM2.5 data in 49 cities with serious PM2.5 pollution in China in 2017. We find that these data are all functional data. First, we fitted these data, and then derives the multivariate functional linear regression model. Finally, we use air pressure and temperature as predictor variables, PM2.5 as response variables, and use a multivariate functional linear regression model to study the effect of air pressure and temperature on PM2.5 when the number of basis functions can be different from each other, then the relevant conclusions are drawn.

When $K_{1}, K_{2}$ change, the value of beta air pressure is positive, which shows that there is a positive correlation between air pressure and PM2.5, but the positive correlation is different. In summer, the air pressure is low and the air has strong convection, which can disperse the PM2.5 pollutant well. Therefore, the content of PM2.5 will be relatively reduced; The higher atmospheric pressure in winter makes most of the pollutants unable to be dispersed, resulting in more PM2.5.

When $K_{1}, K_{2}$ change, the beta temperature is negative. This is quite different from the result of univariate functional linear regression model, and the result is largely due to the collinearity of air pressure and temperature.

\section{Acknowlegements}

Special fund for talents of North China University of Technology (207051360020XN140 / 004)

\section{References}

1. Tucker L R. Determination of Parameters of a Functional Relationship by Factor Analysis [M]. Psychometrika, 23 (1958).

2. J.O.Ramsay, B.W.Silverman. Functional Data
Analysis[M]. Springer Series in statistics. Second ed. Springer, New York (2005).

3. Yan Mingyi. Ideas, methods and applications of statistical analysis of functional data [J]. Statistical Research, 2: 87-94 (2007).

4. Yan Mingyi. Comprehensive evaluation of quality of life: a method based on functional features of data [J]. Statistics and Information Forum, 2: 13-17 (2007).

5. Meng Yinfeng. Method and application of functional data modeling [D]. Shanxi University (2017).

6. Meng Yinfeng. Principal component analysis in functional data analysis [J]. Journal of Shanxi University (Natural Science Edition), 34 (1): 21-25 (2011). 